\author{
Akira Mizuno*
}

\title{
A Brief Review of Interpretation Research in Japan
}

\begin{abstract}
This paper reviews current empirical and theoretical research on interpreting in Japan. Though interpreting research in Japan is in its infancy, it is hoped that it will be activated by the effort of the Interpreting Research Association of Japan. One of the important themes of interpreting research in Japan is to find ways in which to overcome the difficulties that arise in the interpretation between Japanese, which is an SOV-type language, and SVO-type languages such as English.
\end{abstract}

Interpreting research in Japan is still in the early stage of its development. By the end of the 1980's, a small number of researchers, mostly interpreter-researchers who were not in touch with each other, had written some papers on interpreting. They seemed to have had little or no knowledge of the research conducted in the rest of the world. Consequently, their bibliographical references included introductory textbooks of interpretation and writings by practicing interpreters that were neither academic nor scientific. On no occasion did they include theoretical or empirical research done overseas.

With the establishement of the Interpreting Research Association of Japan in 1991, the interpreting research scene in Japan began to change. Though small in membership, the Association has actively engaged in the introduction and translation of interpreting research in other countries. At its regular meetings, members of the Association have reported on important works on interpretation written by such authors as Barik (1969), Paneth (1957), Gerver (1976), Lambert (1983), and González et al. (1991), among others. Some members of the Association have attended the conferences on interpreting and translation held in Brisbane, Australia, in 1993 and in Turku, Finland, in 1994. The virtual seclusion of Japanese researchers on interpreting has finally come to an end.

\footnotetext{
* Akira Mizuno

NHK Bilingual Center

NHK Joho Network

2-2-1 Jinnan, Shibuya-ku

Tokyo 150-01 Japan
}

Hermes, Journal of Linguistics no. 14 - 1995 
Though there has been no discernible 'paradigm' in interpreting research in Japan, communication theory has been a shared concern of Japanese interpreter-researchers. Saito (1991), Kondo (1991), Funayama (1985), Laktorin-Inoue (1991), and the major textbooks on interpretation (Kunihiro, Nishiyama, \& Kanayama 1969; Nihon Tsuyaku Kyokai 1976) all refer to communication theory. However, with the exception of Funayama, who explained the difference between interpreters and bilinguals, and Kondo, who described the three-party two-language model developed by Kirchhoff (1976) and discussed the implications of communication theory on interpreting, it is hard to tell what aspects of interpreting they intended to explain by using communication theory. It is true that interpretation is one mode of communication, but, in view of the fact that interpretation is a complicated and multifaceted phenomenon, communication theory seems to be too crude a tool to account for the variety of aspects posed by interpretation. Communication theory, in short, has only a limited explanatory power. Partly because of this limitation, Japanese researchers until recently rarely dared to attempt to explore the internal processes of interpretation.

\section{Empirical Research}

This paper will first address the empirical research, though small in number, conducted by Japanese researchers.

Kume (1985) reports a case study on consecutive interpretation, in which 15 subjects participated. He analyzed the translation rate and the quality of translation in two modes of consecutive interpretation, a) consecutive interpretation with note-taking and b) consecutive interpretation with manuscript. He concluded that the latter was better than the former in translation rate and that the former was better than the latter in the quality of translation.

Nishio (1986) analyzed an example of simultaneous interpretation from Japanese to English and suggested that the changes in word order and word class, and anticipation play a crucial role in simultaneous interpreting from Japanese to English. The importance of anticipation in that case is almost self-evident if one considers the fact that the basic word order (or, more precisely, the basic constituent order) of the English language is SVO and that of Japanese, SOV. Although Nishio does not refer explicitly to the language-pair-specific issues in sim- 
ultaneous interpreting, she is, in fact, dealing with strategies for coping with the difficulties in simultaneous interpretation between languages with different basic word orders. She concludes that the strategies found in her examples were devised to alleviate the burden on the shortterm memory of the interpreter. She puts it as follows:

"After all, all the tools used in simultaneous interpretation were designed to make maximum use of flexibility in the interpretation of speech within reasonable bounds, while at the same time, reproducing both the thrust and meaning of the original as accurately as possible... In simultaneous interpretation, the techniques used have been designed for the most part to assist the short-term memory of the interpreter, for example, the fairly unrestricted use of changes in syntax." (Nishio 1986, 11)

Similar analysis was conducted on simultaneous interpretation from English to Japanese by Tominaga (1991). He analyzed his own interpretation and found various translation techniques. He says as follows:

"In simultaneous interpretation between English and Japanese interpreters employ certain techniques in order to cope with the time constraint and the significant difference in syntax between the two languages." (Tominaga 1991, 32; my translation)

However, it should be noted that a comparative analysis of a large amount of data would be necessary to verify his findings.

Making use of Tominaga's material, Kondo (1992) examined in great detail how the interpreter translated English postmodifying clauses into Japanese. Simultaneous interpretation of English postmodifying clause into Japanese is one of the most difficult techniques because the Japanese language does not allow postmodification of nouns. There is no exception to this rule. Kondo identified eight strategies that have great pedagogical implications when teaching simultaneous interpreting from English to Japanese.

Nagata (1994) dealt with the case of simultaneous interpreting from Japanese to Chinese. Her main concern was to find whether the presence of manuscript has any effect on simultaneous interpreting. Comparing sight translation with the manuscript and interpretation without manuscript, she examined the time lag between the source text and the target text (measured by the number of chunks) and omissions and additions in translation. She concludes that no significant difference was found between them and hence the presence or non-presence 
of the manuscript has no effect on the performance of interpreters. But she qualifies her conclusion by commenting that an analysis of a large number of samples could produce a significant difference and that the experience of interpreters may have bearing on the result.

Taya (1990) made one of the few studies carried out by psychologists. Through a temporal analysis of a 20-minute simultaneous interpretation from English to Japanese, he considered the characteristics of the human information processing capacity, or 'working memory' in dealing with a continuous input of cognitive workload which requires semantic processing. One unit of Japanese translation was considered to be a verbal protocol, which corresponds to the information retained in the working memory as a unit of meaning immediately before production. He defined a chunk as 'a unit of meaning thought to be grasped by the interpreter' through the comparison of source text and translation. He then counted the number of chunks $(\mathrm{N})$ that are present in the working memory space from the beginning of the translation of one chunk (which is not included in $\mathrm{N}$ ) to its completion. He reports that in $80 \%$ of the total, $\mathrm{N}$ was three or less $(\mathrm{N} \leq 3)$ (total number of chunks in the working memory space $=4$ ), and in $90 \%$ of the total, $N$ was four or less $(\mathrm{N} \leq 4)$ (total number of chunks in the working memory space $=5$ ). Based on the result, he reasoned that interpreters are well aware of the processing capacity of their working memory and they process information so as not to exceed their processing capacity. He further states that the upper limit of $\mathrm{N}$ is around 3 or 4 and infers from the relationship between $\mathrm{N}$ and the number of translation loss that the workload becomes heavier during listening than during production, especially in the case of $\mathrm{N} \leq 3$. He concludes that the processing strategy of interpreters should be to reduce the number of chunks in the working memory space during the listening task. It should be noted, however, that the real question for practicing interpreters is what they should do to reduce the number of chunks in the working memory space during simultaneous interpreting. Partial answers to this question can be found in the papers by Nishio, Tominaga, and Kondo mentioned above.

As a part of a series of studies on reading span, Osaka (1994) examined the correlation between interpreting and the working memory. She characterizes interpretation as a process in which interpreters semantically process incoming sentences, temporarily retain the processed contents as contextual information, and integrate them into the 
next processing. Thus, information retention and processing proceed parallel to each other. The working memory exerts influence on the performance of simultaneous interpretation, which requires the parallel operation of information retention and processing. Based on this assumption, Osaka examined the correlation between the working memory span and interpreting. She conducted listening span tests, memory span tests, shadowing, consecutive interpretation, and simultaneous interpretation using 45 subjects who were undergraduate students majoring in Italian at the Osaka University of Foreign Studies. The result shows a significant correlation between the working memory and consecutive interpretation, whereas no clear statistical difference was found in the case of simultaneous interpretation. Osaka attributes the latter finding to the fact that simultaneous interpreting requires special training.

It was Yoshitomi \& Arai (1991) who conducted experimental research that used by far the largest number of subjects. The total number of subjects was 189 , of whom 154 were undergraduate students in a Foreign Language Department and 34 were trainees studying interpreting in private training schools. Unfortunately, it must be pointed out that the experimental hypothesis of their study includes ambiguous and confusing statements. A few quotations suffice to illustrate this point:

"If one supposes that shadowing exercise is a useful training method for interpreting, it would follow that the longer the training experience the interpreter has and the more skilled the interpreter, the better their shadowing performance. And if the skill of speaking while listening is essential in the interpreting process, the more skilled the interpreter, the better their shadowing performance, regardless of whether they have had shadowing training experience or not." (Yoshitomi \& Arai 1991, 376; my translation)

"We have attempted to verify the effect shadowing exercises have on interpreting skills and at the same time tried to consider shadowing as a yardstick for evaluating trainees." (Yoshitomi \& Arai 1991, 376; my translation)

"The aim of this experiemnt was not to examine the correlation between shadowing skills and interpreting skills, but to look into the correlation between shadowing skills and comprehension which is considered to be at the core of interpreting skills." (Yoshitomi \& Arai 1991, 413; my translation) 
The second quotation is clearly incongruous with the third. What they examined in reality is the correlation between shadowing skills and comprehension. The logical inconsistency of their study could blur the merits of this otherwise laudable work. Though fraught with these defects, the study includes several findings which are of particular relevance to interpretation research. For example, they report that those who are excellent in shadowing also have an excellent comprehension without focusing too much of their attention on the surface structure or the physical sound of the incoming speeches.

Iguchi \& Tanaka (1991) carried out a qualitative analysis of simultaneous interpretation from English to Japanese by comparing the simultaneous interpretation of professional interpreters and trainee interpreters. The number of subjects was 39, of whom two were professional interpreters and 37 were trainee interpreters. They report the following findings among others:

- simultaneous interpretation by professional interpreters includes many conjunctives and interjections

- simultaneous interpretation by professional interpreters includes a lot of rephrasing and complementary expressions

- professional interpreters make an effort to avoid misunderstanding that derive from homonyms by adding corresponding words of the original speech which can be understood by the audience

- professional interpreters try to preserve as much as possible the phrase order or clause order of the source language

Tanaka (1991) made a qualitative error analysis of simultaneous interpretation by 20 trainee interpreters. She analyzed translations rendered by the superior group and the inferior group of the trainee interpreters and reports that the interpretation by a superior group includes more complete sentences and less silence than that of an inferior group. Based on this finding, she speculates that the superior group has a firm grasp of contextual meaning whereas the inferior group tends to focus too much attention to the surface structure of the source text. She further reports that the ear-voice span (the time lag between the beginning of original speech and the beginning of its translation) of the inferior group is longer than that of the superior group, thus further straining the limited processing capacity. She attributes the longer earvoice span of the inferior group to their lack of translation techniques 
that would enable them to circumvent the overloading of the short-term memory.

\section{Theoretical Studies}

Though there are several textbooks on interpretation and books written by practicing interpreters on the profession and the process of interpretation, there are few theoretical accounts on the mechanisms and processes of interpretation.

In a paper that dealt mainly with speaking, Kohno \& Miyake (1985) briefly touched upon simultaneous interpretation. They tried to give a rationale to what makes simultaneous interpretation possible by using the dichotomy of 'voluntary utterance' and 'automatic utterance.' They put it as follows:

"(In simultaneous interpretation from English to Japanese,) in order to produce a Japanese translation, interpreters require a short duration of time during which they listen attentively to the source language without uttering a word. This operation is a highly cognitive task. But once they decide what to say (as translation) and begin uttering the Japanese translation, the operation turns into a kind of reflex, with the result that the phonological regulations and the manipulation of postpositional particles or inflexions are carried out automatically." (Kohno \& Miyake 1985, 97; my translation)

They say that "one cannot carry out two or more cognitive tasks simultaneously in language processing."

Another explanation of the process of simultaneous interpretation was presented by Hagiwara (1986). Based on his experience of teaching listening comprehension to university students, he claims that the construction of 'fusion' or 'Verschmelzung' (i.e. the concept or semantic representation shared by the source language and the target language) is impossible for Japanese university students with the exception of clichés. He says that the listening comprehension of Japanese university students is nothing but a rapid internal (mental) translation which shares various characteristics with simultaneous interpretation. He maintains that the listening, the rapid internal (mental) translation, short-term retention of verbal material, and verbal production are carried out parallel to each other during simultaneous interpretation and the first three operations are common to the listening comprehension of 
Japanese students. He further discussed cognitive, psychological, and physiological aspects of simultaneous interpretation, consecutive interpretation, and listening comprehension. He may not have known Seleskovitch (1978), but his claim that 'fusion' is impossible in simultaneous interpreting is in stark contrast to the 'deverbalization' of her theory of sense.

One of the interpreter-researchers who dealt with various aspects of simultaneous interpretation is Funayama (1985). He summarizes communicative, linguistic, technical, and vocational aspects of simultaneous interpretation in order to delineate the whole picture of simultaneous interpretation. In his recent papers (Funayama 1993 and 1994), he tried to explain the seemingly unique phenomena which appear during simultaneous interpreting by using the concept of 'cognitive file' first developed by J. W. DuBois and W. Croft. The concept of cognitive file is set at the semantic level and provides a context in discourse, with the function of sustaining discourse cohesion. Funayama's cognitive file differs from that of DuBois and Croft because his concept is set closer to the language level, whereas the concept of DuBois and Croft is used only with regard to referents. The phenomena that suggest the presence of cognitive file in simultaneous interpretation are as follows: - the multiple appearance of a source language lexical unit in the target language

- some cases of non-equivalent expressions

- division and synthesis of source language concept(s) in the target language

- long retention of information on the part of the interpreter

Funayama suggests that the cognitive file would be useful to explain not only the above-mentioned phenomena but also the comprehension process. His study will contribute to the elucidation of the memory structure and discourse cohesion in simultaneous interpretation.

Arai (1991) reviewed Western literature on interpretation and language communication. However, probably because of the difficulty in locating material, the selection of literature is arbitrary and biased. She refers to Seleskovitch several times but does not mention the important works by researchers at the Scuola Superiore di Lingue Moderne per Interpreti e Traduttori of the University of Trieste. In the section on theoretical studies and cognitive psychology, she makes no reference to 
the information processing models proposed by Gerver (1976) and by Moser (1978). It seems quite strange in view of the fact that in her review she mentions other articles from the same books, Brislin (1976) and Gerver \& Sinaiko (1978), which include the above-mentioned articles by Gerver and Moser.

On the methodological aspects of interpretation research, Mizuno (1993a), while supporting the observational and scientific research programs proposed by Gile (1989a, 1989b, and 1991), claims that the induction and hypothetico-deductive method are not enough to investigate the interpretation process and that the criteria of 'falsifiability' proposed by Popper (1959) and the investigative methods in the field of cognitive science should be employed in interpretation research. He takes the view that a testable hypothesis needs a theoretical framework that would provide a general explanation of the interrelationships between complex phenomena. Induction by observation is not the only way to construct a theory. What is important is whether the theory can produce testable hypotheses and whether it can be falsified.

Lastly, Mizuno (1993b and 1994) proposed a Dynamic Model of simultaneous interpreting through a critical review of the preceding information processing models of simultaneous interpreting. He examined the theories of Gerver (1976), Moser (1978), Gile (1985 and 1988), Goldman-Eisler (1980), and Lambert (1992) to learn how they solved the problem of concurrent multiple-task operations during simultaneous interpreting. Based on the concepts of 'automatic and controlled processes' proposed by Schneider \& Shiffrin (1977) and Shiffrin \& Schneider (1977), he rejects the idea of 'divided attention' (Gerver 1976) which claims that attention can be easily divided between various functions under normal circumstances. He also argues that, within the limit of processing capacity, a combination of controlled process, partially-automatic process, and automatic process enables the performance of concurrent tasks required in simultaneous interpretation.

\section{Interpretation and Culture}

Among the works on the relationship between interpreting and sociocultural factors, the first to be noted is Kondo (1988). He explored the socio-cultural factors that might affect the social status of Japanese interpreters. Arguing that the homogeneity of the overwhelming major- 
ity of the Japanese people plays an important role in determining the way they communicate, he describes the characteristics of Japanese communication style as follows:

- little need to explain oneself explicitly

- little need for overt verbal communication

- scorn for those who fail to understand subtle communication

- scorn for those who use many words to communicate

In Japanese society where silence is golden and "a smooth flow of words would give an impression of superficiality and insincerity," interpreters are likely to be considered "mere hearing and speaking machines" (Kondo 1988, 71). Kondo further comments that partly because of these circumstances the interpreters' status in Japan is low "economically, socially, culturally and even intellectually" (Kondo 1988, 71).

In a short paper, Hara (1988) dealt with the linguistic as well as the socio-cultural problems of Japanese language as a tool for transcultural communication. Though her arguement is rather vague with respect to its socio-cultural implications, she points out that the sentence structure that puts the verb right at the end of the sentence, the presence of multitudes of homophones, and the complicated system of honorifics present a great challenge for intercultural communication.

More recently, Torikai (1994) took up two cases of intercultural miscommunication: a meeting of President Nixon and Prime Minister Sato in 1971 and the Japanese Government's response to the Potsdam Declaration in 1945 . Though Torikai does not explicitly blame one party or the other for those intercultual miscommunications, the root cause of the miscommunications may be attributed to the ambiguous expressions and the insensitivity to intercultural communication on the part of Mr. Sato and the Japanese government. Torikai concludes that interpreters put in those circumstances should be given sufficient background information so that they may choose contextually appropriate words and expressions in the target language.

\section{Concluding Remarks}

With the increasing interaction between Japanese researchers and their colleagues overseas, future research on interpreting in Japan cannot continue independent from and ignorant of research done overseas. 
Japanese researchers will be turning to study common issues with researchers in foreign countries. Through this short review, however, one may discern a shared concern of Japanese interpreter-researchers. That is to find ways to cope with the difficulties that arise from the difference of syntax (basic constituent order) in interpreting between SVO-type languages and Japanese. One of the future research directions will involve this issue and it is hoped that it will contribute to research on interpretation between SVO-type languages and SOV-type languages in general.

\section{References}

Arai, Kiwa (1991): Tsuyaku ni kansuru Bunken Kenkyu (Literature Review on Interpretation). In: Watanabe 73-87.

Barik, Henri, C. (1969): A Study of Simultaneous Interpretation. Ph.D. Thesis. University of North Carolina.

Funayama, Chuta (1985): Dojitsuyaku no Shosokumen (Aspects of Simultaneous Interpretation). In: Shichokaku Gaikokugo Kyoiku Kenkyu (Bulletin of the Language Laboratory) 8, 49-66.

Funayama, Chuta (1993): Danwa-kessokusei no tame no Ninchi Failu (Cognitive Files for Discourse Cohesion). In: Ei-bei Gengo Bunka Kenkyu (British and American Language and Culture) 41, 89-101.

Funayama, Chuta (1994): Dojitsuyaku ni Arawareru Ninchi Failu (Cognitive Files that Appear during Simultaneous Interpreting). In: Jiji Eigogaku Kenkyu (Current English Studies) 33, 1-12.

Gerver, David (1976): Empirical Studies of Simultaneous Interpretation: A Review and a Model. In: R. Brislin (Ed.) Translation: Applications and Research. New York: Gardner Press.

Gile, Daniel (1985): Le Modèle d'efforts et l'équilibre d'interprétation simultanée. In: META 30: 1, 44-48.

Gile, Daniel (1988): La Partage d'attention et le 'modele d'effort' en interprétation simultanée. In: Interpreters' Newsletter 1, 4-22.

Gile, Daniel (1989a): Scientific Research vs. Personal Theories in the Investigation of Interpretation. In: Gran, Laura \& Taylor, Christopher (Eds.): Aspects of Applied and Experimental Research on Conference Interpretation: Round Table on International Research. Udine: Campanotto Editore.

Gile, Daniel (1989b): Research Proposals for Interpreters. In: Gran, Laura \& Taylor, Christopher (Eds.): Aspects of Applied and Experimental Research on Conference Interpretation: Round Table on International Research. Udine: Campanotto Editore.

Gile, Daniel (1991): Methodological Aspects of Interpretation (and Translation) Research. In: Target 3: 2, 153-174. 
Goldman-Eisler, F. (1980): Psychological Mechanisms of Speech Production as Studied through the Analysis of Simultaneous Translation. In: Butterworth, B. (Ed.) Language Production Vol.1 Speech and Talk. London: Academic Press.

González, R. D. \& Vásquez, V. F. \& Mikkelson, H. (1991): Fundamentals of Court Interpretation: Theory, Policy, and Practice. Durham: Carolina Academic Press.

Hagiwara, Naoyuki (1986): Daigaku Eigo Chokai Kunren he no Doji-Chikuji Tsuyaku (teki) Gihou no Donyu. (Introducing Interpreting Techniques in Listening Comprehension Training for University Students). In: Hasegawa, Kiyoshi \& Sumitomo, Koichi (Eds.) Hoso Eigo no Riyouhou. Tokyo: Taishukan.

Hara, Fujiko (1988): Understanding the Silent Culture of the Japanese. In: META 33: $1,22-24$.

Iguchi, Sanae \& Tanaka, Makiko (1991): Puro no Tsuyakusha oyobi Tsuyaku Kunrensei no Tsuyaku no Yakushutsujo no Tokucho. (A Comparison of Performance of Professional Interpreters and Student Interpreters). In: Watanabe, 143-237.

Kirchhoff, H. (1976): Das Dreigliederige, Zweisprachige Kommunikationssystem Dolmetschen. In: Le Langage et l'Homme, May 1976.

Kohno, Morio \& Miyake, Yutaka (1985): Speaking no Mekanizumu - Listening to no Kankei (Speaking Mechanism: Its Relationship to Listening). In: Kohno, Morio \& Sawamura, Fumio (Eds.) Listening \& Speaking: Atarashii Kangaekata. Kyoto: Yamaguchi Shoten.

Kondo, Masaomi (1988): Japanese Interpreters in Their Socio-Cultural Context In: META 33: 1, 64-69.

Kondo, Masaomi (1991): Tsuyakuno San-sha Ni-gengo Modelu (Three-Party TwoLanguage Model of Interpreting). In: Interpreting Research 1: 1, $23-24$.

Kondo, Masaomi (1992): Eigo ni okeru Kochi Shushokusetsu no Honyaku Dojitsuyaku no Shorihou yori Manabu (How English Postmodifying Clauses Can be Better Translated into Japanese - Lessons from Simultaneous Interpreting Techniques). In: Daito Bunka Daigaku Kiyou - Humanities 30, 277-294.

Kume, Akimoto (1985): Chikuji Tsuyaku he no Apurochi - Nihongo Yakushutsu ni okeru Hikaku Kenkyu (An Approach to Consecutive Interpretation: A Case Study of Japanese Translation). In: Jiji Eigogaku Kenkyu. (Current English Studies) 24, 23-34.

Kunihiro, Masao \& Nishiyama, Sen \& Kanayama, Nobuo (1969): Tsuyaku - Eikaiwa kara Dojitsuyaku made (Interpreting: From English Conversation to Simultaneous Interpretation). Tokyo: Nihon Hoso Shuppan Kyokai.

Laktorin-Inoue, Kumi (1991): Ningen Kyoiku to shite no Tsuyakusha Kyoiku (Interpreter Training as Human Education). In: Watanabe, 27-65.

Lambert, Silvie (1983): Recall and Recognition among Conference Interpreters. Ph. D. Thesis, University of Stirling.

Lambert, Silvie (1992): Shadowing. In: Interpreters' Newsletter. 4, 15-24. 
Mizuno, Akira (1993a): Tsuyakusha ni yoru Tsuyaku Kenkyu no Houhou (Methodological Issues of I/T Research by Interpreters). In: Interpreting Research 3: 2, 29-38.

Mizuno, Akira (1993b): Dojitsuyaku no Dotai Modelu ni Mukete (Toward a Dynamic Model of Simultaneous Interpreting). Paper read at IJET4.

Mizuno, Akira (1994): Dojitsuyaku Dotai Modelu no Tenkai (I) (The Dynamic Model of Simultaneous Interpretation (I)). In: Interpreting Research. 4: 2, 13-25.

Moser, Barbara (1978): Simultaneous Interpretation: A Hypothetical Model and Its Practical Application. In: Gerver, David \& Sinaiko, H. Wallace (Eds.) Language Interpretation and Communication. New York and London: Plenum Press.

Nagata, Sae (1994): Nihongo kara Chugokugo he no Dojitsuyaku Bunseki. In: Interpreting Research 4: 2, 9-12.

Nihon Tsuyaku Kyokai (Ed.) (1976): Tsuyaku Kyohon: Eigo-Tsuyaku he no Michi (Textbook of Interpretation: The Way to English Interpreting). Tokyo: Taishukan.

Nishio, Michiko (1986): A Brief Introduction to the Mechanics of Simultaneous Interpretation with Special Reference to Japanese-English Interpretation. In: The Language Teacher 10: 2, 4-12.

Osaka, Mariko (1994): Riidingu Supan no Kenkyu (5) - Dojitsuyaku Tesuto to Waakingu Memori (The Study of Reading Span (5): Simultaneous Interpreting Tests and Working Memory). In: Nihon Shinri Gakkai Dai-58-Kai Taikai Ronbunshu (Proceedings of the 58th Convention of the Japan Psychological Society). 710.

Paneth, E. (1957): An Investigation into Conference Interpreting (with Special Reference to the Training of Interpreters). M. A. Degree in Education to the University of London

Popper, Karl R. (1959): The Logic of Scientific Discovery. London: Hutchinson.

Saito, Fukunaga Mitsuko (1991): Tsuyaku Gijutsu to Riron no Kochiku (Establishing Interpreting Techniques and Theory). In: Watanabe: 449-764.

Schneider, Walter \& Shiffrin, Richard M. (1977): Controlled and Automatic Information Processing: I. Detection, Search, and Attention. In: Psychological Review 84: 1, 1-66.

Seleskovitch, D. (1978): Interpreting for International Conferences: Problems of Language and Communication. Washington, D. C.: Pen and Booth.

Shiffrin, Richard M. \& Schneider, Walter (1977): Controlled and Automatic Information Processing: II. Perceptual Learning, Automatic Attending, and a General Theory. In: Psychological Review 84: 2, 127-190.

Tanaka, Makiko (1991): Tsuyaku Kunrensei no Dojitsuyaku ni okeru Goyaku Bunseki to Kousatsu (Error Analysis of Simultaneous Interpretation Rendered by Student Interpreters). In: Watanabe: 161-237.

Taya, Norihiko (1992): Dojitsuyaku ni okeru Yakushutsu Jokyo Bunseki (An Analysis of Interpreting Process in Simultaneous Interpreting). In: Nihon Shinri Gakkai Dai56-Kai Ronbunshu (Proceedings of the 56th Convention of the Japan Psychological Society) 628 . 


\section{4}

Tominaga, Masayuki (1991): Ei-Nichi Dojitsuyaku ni Miru Yakushutsu Shori Houhou (English-Japanese Simultaneous Interpreting Techniques). In: Interpreting Research 1: 1, 32-35.

Torikai, Kumiko (1994): Tsuyaku to Honyaku - Ibunka no Hashiwatashi (Interpretation and Translation: Bridging Cultural Gaps). In: Honna, Nobuyuki \& Akiyama, Kohji \& Takeshita, Yuko \& Hoffer, Bates (Eds.) Ibunka Rikai to Komyunikeeshon 1 (Cross-Cultural Understanding and Communication 1). Tokyo: Sanshusha.

Watanabe, Shoichi (Ed.) (1991): Monbusho Josei Kagaku Kenkyu: Gaikokugo Kyoiku no Ikkan to shite no Tsuyaku Yosei no tame no Kyoiku Naiyou Houhou no Kaihatsu ni kansuru Sogo-teki Kenkyu (A Comprehensive Study on the Development of Interpretation Training Methodology as a Part of Foreign Language Training). Report on a Project funded by the Japanese Ministry of Education. Published in March 1991 by the Office of Prof. Matsuo of Sophia University, Tokyo.

Yoshitomi, Asako \& Arai, Kiwa (1991): Tsuyaku Katei ni kansuru Kiso Jikken (A Basic Experiment on the Interpreting Process). In: Watanabe: 373-439. 